\title{
Does Good Mutation Help You Live Longer?
}

\author{
W. Hwang, P. L. Krapivsky, and S. Redner \\ Center for BioDynamics, Center for Polymer Studies, and Department of Physics, Boston University, Boston, MA 02215
}

\begin{abstract}
We study the dynamics of an age-structured population in which the life expectancy of an offspring may be mutated with respect to that of its parent. When advantageous mutation is favored, the average fitness of the population grows linearly with time $t$, while in the opposite case the average fitness is constant. For no mutational bias, the average fitness grows as $t^{2 / 3}$. The average age of the population remains finite in all cases and paradoxically is a decreasing function of the overall population fitness.
\end{abstract}

In this letter, we investigate the role of mutation on the age distribution and fitness of individuals within a simple age-structured population dynamics model. The basic feature of our model is that the life expectancy of an offspring, which measures its fitness, may be mutated with respect to that of its parent. While age-structured population models have been studied previously [1,2], relatively little is known about the role of mutation. When the individual reproduction rate is the fitness measure and population is regulated by externally imposed death, mutation leads to predominance by the fittest species [3]. In a related vein, it was recently shown that longevity is heritable [4,5] within the Penna bit-string model of aging [6]. In these studies, the role of positive mutations was central. Our focus is quite different, as we study the dynamics of the fitness and age in a self-interacting population as a function of the advantageous and deleterious mutation rates.

When advantageous mutation is favored, that is, the offspring life expectancy (fitness) is greater than that of its parent, the fitness distribution of the population approaches a Gaussian with average fitness growing linearly in time and dispersion increasing as $t^{1 / 2}$. Conversely, when deleterious mutation is more likely, there is a $t^{-2 / 3}$ approach to a steady fitness distribution. In the absence of mutational bias, the fitness distribution again approaches a Gaussian, with average fitness growing as $t^{2 / 3}$ and width growing as $t^{1 / 2}$. The average age of the population reaches a steady value in all cases and, surprisingly, is a decreasing function of the average fitness. Therefore within our model, a fitter population leads to a decreased individual lifetime.

Our model is a simple population dynamics scenario which incorporates age structure and mutation. This dynamics is based on the logistic model, $\dot{N}=b N-\gamma N^{2}$, in which a population with density $N(t)$ evolves both by birth at rate $b$, and death at rate $\gamma N$, with steady-state solution $N_{\infty}=b / \gamma$. The crucial new element in our model is that the life expectancy of each newborn may be mutated by $\pm \tau$ (with $|\tau|=1$ without loss of generality) with respect to that of its parent. We also assume a constant age-independent mortality rate and birth rate for each individual.

Each of these features represent idealizations of reality; for example, it would be more realistic to incorporate a mortality rate which is an increasing function of age
[2,5:4]. We shall argue below that our choice of an ageindependent mortality leads to behavior which applies to systems with realistic mortality rates. The nature of our results also suggests that the details of the mutationdriven shift in offspring life expectancy is not crucial.

Let $C_{n}(a, t)$ be the density of individuals with life expectancy $n \geq 1$ and age $a$ at time $t$. According to our model, the rate equation for $C_{n}(a, t)$ is

$$
\left(\frac{\partial}{\partial t}+\frac{\partial}{\partial a}\right) C_{n}(a, t)=-\left(\gamma N(t)+\frac{1}{n}\right) C_{n}(a, t) .
$$

The derivative with respect to $a$ on the left hand side accounts for aging [1, 8]. On the right hand side, the loss term $\gamma N C_{n}$ accounts for death by competition and is assumed to be independent of an individual's age and fitness. As discussed above, the mortality rate is taken as age independent; the form $C_{n} / n$ guarantees that the life expectancy equals $n$.

We account for the population of newborns as a boundary condition for $C_{n}(a=0, t)$ [1]. An individual produces offspring with the same life expectancy at rate $b$, and, due to mutation, produces offspring whose life expectancy is longer or shorter than its parent by \pm 1 , with respective rates $b_{ \pm}$. Defining $P_{n}(t)=\int_{0}^{\infty} d a C_{n}(a, t)$ as the density of individuals at time $t$ of any age whose life expectancy equals $n$, then the boundary condition for $C_{n}(0, t)$ is

$$
C_{n}(0, t)=b P_{n}(t)+b_{+} P_{n-1}(t)+b_{-} P_{n+1}(t) .
$$

To determine the asymptotic behavior of the age and fitness distributions, it proves useful to first disregard the age structure and focus on fitness alone. From Eqs. (1) (2), the rate equations for $P_{n}(t)$ for $n \geq 1$ are

$$
\frac{d P_{n}}{d t}=\left(b-\gamma N-\frac{1}{n}\right) P_{n}+b_{+} P_{n-1}+b_{-} P_{n+1},
$$

with $P_{0}=0$. This describes a random-walk-like process in a one-dimensional fitness space which is augmented by birth and death due to the first term on the righthand side. Using $N(t)=\Sigma P_{n}(t)$, we find that the total population density obeys a generalized logistic equation

$$
\frac{d N}{d t}=(B-\gamma N) N-\sum_{n=1}^{\infty} \frac{P_{n}}{n}-b_{-} P_{1}
$$

where $B \equiv b+b_{+}+b_{-}$is the total birth rate. 
We now discuss the asymptotic behavior of these rate equations for three basic cases: subcritical - deleterious mutations favored $\left(b_{-}>b_{+}\right)$; critical - no mutational bias $\left(b_{+}=b_{-}\right)$; and supercritical - advantageous mutations favored $\left(b_{+}>b_{-}\right)$. In all three cases, the total population density $N$ and the average age $A=N^{-1} \sum A_{n}$, with $A_{n}=\int a C_{n}(a) d a$, approach steady values. These are determined by a balance between the total birth rate $B$ and the death rate $\gamma N$ due to overcrowding. In the critical and supercritical cases, this leads to the steady state behaviors for the total density and the average age,

$$
N=\frac{B}{\gamma}, \quad A=\frac{1}{\gamma N}=\frac{1}{B} .
$$

The behavior in the subcritical case is more subtle, as we now discuss.

Subcritical Case. Here a steady state is reached whose properties are found by setting $\frac{d P_{n}}{d t}=0$ in Eq. (3). We solve this rate equation by introducing the generating function $F(x)=\sum_{n \geq 1} P_{n} x^{n-1}$ to transform the rate equation into the differential equation

$$
\frac{F^{\prime}}{F}=\frac{\gamma N-b+1-2 b_{-} x}{b_{-}-(\gamma N-b) x+b_{+} x^{2}} .
$$

Integrating Eq. (6), subject to the obvious boundary condition $F(1)=N$, gives a family of solutions which are parameterized by the total population density $N$. To extract a unique solution one has to invoke on additional arguments. First notice that $N$ lies within a finite range. The upper limit is found from the steady-state version of Eq. (4),$(B-\gamma N) N=\Sigma n^{-1} P_{n}+b_{-} P_{1}>0$, to give $\gamma N<B$. The lower limit is obtained from the physical requirement that all the $P_{n}$ 's are positive and therefore $F(x)$ is an increasing function of $x$. From Eq. (6) this leads to the inequality $(\gamma N-b)^{2} \geq 4 b_{+} b_{-}$. Thus

$$
b+2 \sqrt{b_{+} b_{-}} \leq \gamma N<B .
$$

For any initial condition for the $P_{n}$ with a finite support in $n$, only the minimal solution which satisfies the lower bound of Eq. (7) is realized. This selection is reminiscent of the behavior in the Fisher-Kolmogorov equation and related reaction-diffusion systems [1].

To understand why the minimal solution is selected, consider the steady-state asymptotic behavior of $P_{n}$ for $n \rightarrow \infty$. In this limit, we may neglect the $P_{n} / n$ term in Eq. (3). The resulting quasi-linear equation has the solution $P_{n}=A_{+} \lambda_{+}^{n}+A_{-} \lambda_{-}^{n}$, with $\lambda_{ \pm}=$ $\left[\gamma N-b \pm \sqrt{(\gamma N-b)^{2}-4 b_{+} b_{-}}\right] / 2 b_{-}$, and with $\lambda_{ \pm}<$ 1. Thus the steady-state fitness distribution decays exponentially with $n$. When the total population density attains the minimal value $N_{\min }=\left(b+2 \sqrt{b_{+} b_{-}}\right) / \gamma, \lambda_{+}$ achieves its minimum possible value $\lambda_{+}^{\min }=\sqrt{b_{+} / b_{-}} \equiv$ $\mu^{-1}$, where $\mu$ is the mutational bias. Since $P_{n} \sim \lambda_{+}^{n}$, the fitness distribution has the most rapid decay in $n$ for the minimal solution. This minimal solution appears to be the basin of attraction for any initial condition with $P_{n}(0)$ decaying at least as fast as $\mu^{-n}$. Conversely, an initial condition which decays as $\alpha^{n}$ with $\alpha$ in the range $\left(\mu^{-1}, \lambda_{+}^{\max }=1\right)$ should belong to the basin of attraction of the solution where, from the steady-state version of Eq. (3) in the large- $n$ limit, the total population density is $N=\left(b+b_{-} \alpha+b_{+} \alpha^{-1}\right) / \gamma$. We have verified this general classification of solutions numerically [9].

Since the the steady state is approached exponentially in time for the classical logistic equation, $\dot{N}=b N-\gamma N^{2}$, one might anticipate a similar relaxation for our agestructured logistic equation (何. However, a numerical integration of the rate equations gives a power-law relaxation of the total population density, $N_{\infty}-N(t) \sim t^{-2 / 3}$, for a compact initial condition (Fig. 1). This is also verified by an asymptotic analysis of the rate equations [9. A similar relaxation also occurs for the subpopulation densities with given fitness, $P_{n}(t)$.

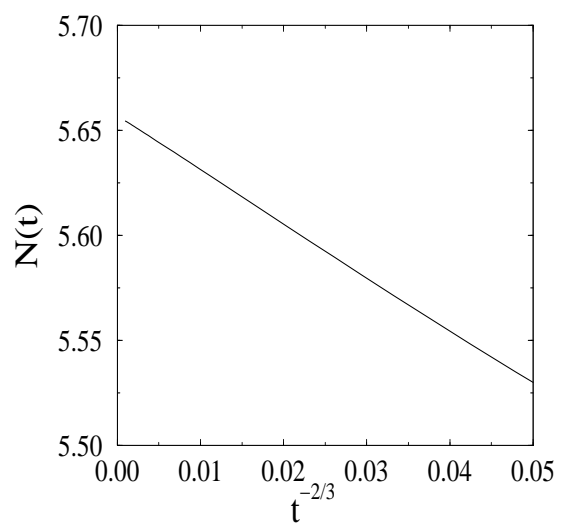

FIG. 1. $N(t)$ versus $t^{-2 / 3}$ in the subcritical case with $b=0$, $b_{+}=1, b_{-}=2$, and $\gamma=0.5$, and with the initial condition $P_{n}(t=0)=0.1$ for $1 \leq n \leq 10$. The asymptotic intercept with the $y$ axis gives the theoretically predicted value of $N_{\min }=4 \sqrt{2} \approx 5.6568$.

For the relevant situation where the density $N$ takes the minimal value, we integrate Eq. (6) to give the generating function

$$
F(x)=N\left(\frac{\mu-1}{\mu-x}\right)^{2} \exp \left\{\frac{x-1}{b_{+}(\mu-x)(\mu-1)}\right\} .
$$

One can formally determine the $P_{n}$ by expanding $F(x)$ in a Taylor series. However, the asymptotic characteristics of the fitness distribution are more easily determined directly from the generating function by using $\left\langle n^{k}\right\rangle=\frac{1}{N} \sum_{n=1}^{\infty} n^{k} P_{n}=\left.\frac{1}{N}\left(x \frac{d}{d x}\right)^{k} F(x)\right|_{x=1}$. Applying this to Eq. (8), the first two moments of the fitness distribution are

$$
\begin{aligned}
& \langle n\rangle=\frac{1}{b_{+}(\mu-1)^{2}}+\frac{2}{\mu-1}+1 \\
& \sigma^{2}=\left\langle n^{2}\right\rangle-\langle n\rangle^{2}=\frac{\mu+1}{b_{+}(\mu-1)^{3}}+\frac{2 \mu}{(\mu-1)^{2}} .
\end{aligned}
$$


The average age of the population may be obtained by first solving Eq. (1) in the steady state to give

$$
C_{n}(a)=P_{n}\left(\gamma N+\frac{1}{n}\right) \exp \left[-\left(\gamma N+\frac{1}{n}\right) a\right]
$$

The average age then is [9]

$$
\begin{aligned}
A & =\frac{1}{N} \sum_{n=1}^{\infty} \int_{0}^{\infty} a C_{n}(a) d a, \\
& =\frac{1}{\gamma N}-\frac{1}{N} \frac{1}{(\gamma N)^{2}} \sum_{n=1}^{\infty} \frac{P_{n}}{n+(\gamma N)^{-1}}, \\
& =\frac{1}{\gamma N}-\frac{1}{N} \frac{1}{(\gamma N)^{2}} \int_{0}^{1} x^{\frac{1}{\gamma N}} F(x) d x,
\end{aligned}
$$

where the second line is obtained by using the expression for $C_{n}(a)$ from Eq. (10) and the last line follows by expressing the sum in terms of an integral of the generating function. The surprising feature that emerges by numerical evaluation of this integral (Fig. 2) is that the average age decreases as the population gets fitter!

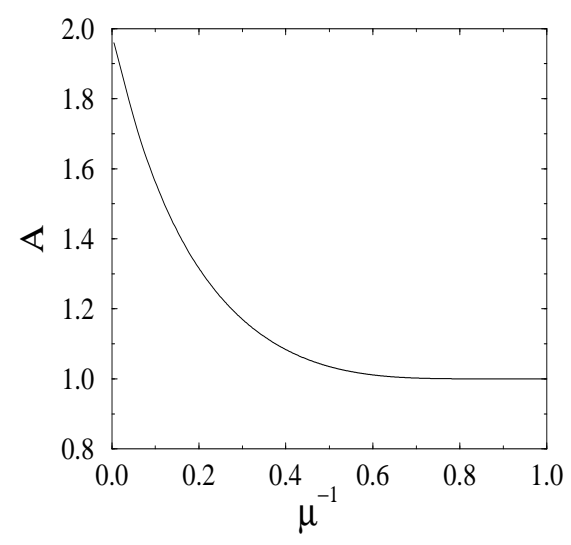

FIG. 2. Average age $A$ of the steady state population versus $\mu^{-1}=\sqrt{b_{+} / b_{-}}$with $b=0$, and with fixed total birth rate equal to one. For $\mu^{-1}>1$, the average age $A=1$, while for $\mu^{-1} \rightarrow 0, A \rightarrow 2$.

Supercritical Case. When $b_{+}>b_{-}$, the random walk in fitness space defined by Eq. (3) is biased away from the origin and a continuum approach becomes appropriate in the long-time limit. Treating $n$ as continuous and Taylor expanding the master equation for small deviations about $n$, gives the following convection-diffusion equation, supplemented by birth/death terms, for the fitness distribution

$$
\left(\frac{\partial}{\partial t}+V \frac{\partial}{\partial n}\right) P=\left(B-\gamma N-\frac{1}{n}\right) P+D \frac{\partial^{2} P}{\partial n^{2}}
$$

The difference between the advantageous and deleterious mutation rates now defines a bias velocity $V \equiv b_{+}-b_{-}$, and the average mutation rate plays the role of a diffusion constant $D \equiv\left(b_{+}+b_{-}\right) / 2$. Integrating over all fitness values, the total population density obeys

$$
\frac{d N}{d t}=(B-\gamma N) N-\int_{0}^{\infty} \frac{P(n, t)}{n} d n .
$$

Since the fitness distribution is sharply peaked at $\langle n\rangle=$ $V t$ (see below), the integral on the right-hand side approaches $N / V t$. By setting $\frac{d N}{d t}=0$ in the resulting equation, we conclude that $\gamma N \rightarrow B-\frac{1}{V t}$. This gives both the steady-state density, as well as the rate of convergence to the steady state.

We now find the fitness distribution by substituting this asymptotics for $N(t)$ into Eq. (12) to give

$$
\left(\frac{\partial}{\partial t}+V \frac{\partial}{\partial n}\right) P=\left(\frac{1}{V t}-\frac{1}{n}\right) P+D \frac{\partial^{2} P}{\partial n^{2}} .
$$

The birth/death term on the right hand side may be neglected, since $\langle n\rangle=V t$, and fluctuations about this average are of order $t^{1 / 2}$. This approximation reduces Eq. (14) into the classical convection-diffusion equation, with solution

$$
P(n, t)=\frac{N}{\sqrt{4 \pi D t}} \exp \left[-\frac{(n-\langle n\rangle)^{2}}{4 D t}\right]
$$

This gives a localized fitness distribution with average fitness growing linearly in time, $\langle n\rangle=V t$, and width growing diffusively, $\sigma=\sqrt{2 D t}$.

To determine the age characteristics, notice that asymptotically, the $P_{n}$ 's change slowly with time, so that the time variable $t$ is slow. On the other hand, the age variable $a$ is fast. Physically this reflects the fact that during the lifetime of a typical individual the change in the age characteristics of the population is small. Thus in the first approximation, we retain only the age derivative in Eq. (1). We also ignore the term $C_{n} / n$, which is small near the peak of the asymptotic fitness distribution. Solving the resulting master equation and using the boundary condition of Eq. (2) we obtain

$$
\begin{aligned}
C_{n}(a, t) & \simeq P_{n}(t) \gamma N e^{-\gamma N a} \\
& =\frac{\gamma N^{2}}{\sqrt{4 \pi D t}} \exp \left[-\gamma N a-\frac{(n-V t)^{2}}{4 D t}\right] .
\end{aligned}
$$

Summing over the fitness variable, the total age distribution $C(a, t)=\sum C_{n}(a, t)$ is just a (stationary) Poisson, $C(a, t)=\gamma N^{2} e^{-\gamma N a}$, and the average age is $A=(\gamma N)^{-1}=B^{-1}$, in agreement with Eq. (5).

Let us compare this average age to that in the subcritical case; the latter is given by Eq. (11) with $\gamma N=$ $b+2 \sqrt{b_{+} b_{-}}$. To provide a fair comparison (Fig. 2), take the total birth rate $B$ to be the same in both cases. It can then be proved that the average age in the supercritical case is always smaller than that in the subcritical case [9]. Individuals in a population with preferential deleterious mutations live longer than if advantageous mutations are favored! The continuous "rat-race" to increased fitness 
in the supercritical case does not lead to an increase in the average life span.

Critical Case. With no mutational bias, the fitness still grows indefinitely, but more slowly than in the supercritical system. The equation of motion for $P(n, t)$ is again given by Eq. (12), but with $V$ set equal to zero and with $N(t)$ is still described by Eq. (13). To derive the scaling behaviors of $\langle n\rangle$ and the width of the fitness distribution, we first use the fact that numerical integration of Eq. (12) again gives a localized fitness distribution. Thus we may estimate the integral on the right-hand side of Eq. (13) as $N /\langle n\rangle$. This leads to $\gamma N \rightarrow B-\frac{1}{\langle n\rangle}$. Substituting this into Eq. (12) for $P(n, t)$ now yields

$$
\frac{\partial P}{\partial t}=\left(\frac{1}{\langle n\rangle}-\frac{1}{n}\right) P+D \frac{\partial^{2} P}{\partial n^{2}} .
$$

To determine the long-time behavior of this equation, we exploit the fact that the fitness distribution is peaked near $n \approx\langle n\rangle$. This suggests changing variables from $(n, t)$ to the co-moving co-ordinates $(y=n-\langle n\rangle, t)$. Eq. (17) then becomes

$$
\frac{\partial P}{\partial t}-\frac{d\langle n\rangle}{d t} \frac{\partial P}{\partial y}=\frac{y}{\langle n\rangle^{2}} P-\frac{y^{2}}{\langle n\rangle^{3}} P+D \frac{\partial^{2} P}{\partial y^{2}} \text {. }
$$

Let us first assume that the average fitness grows faster than diffusively, that is, $\langle n\rangle \gg \sqrt{t}$. With this assumption, the dominant terms in Eq. (18) are

$$
\frac{d\langle n\rangle}{d t} \frac{\partial P}{\partial y}=-\frac{y}{\langle n\rangle^{2}} P .
$$

These terms balance when $\langle n\rangle /(t y) \sim y /\langle n\rangle^{2}$. Using this scaling in Eq. (18) and then balancing the remaining subdominant terms gives $y \sim \sqrt{t}$. The combination of these results then give $\langle n\rangle \sim t^{2 / 3}$. This justifies our initial assumption, $\langle n\rangle \gg \sqrt{t}$. Finally, writing $\langle n\rangle=(u t)^{2 / 3}$, simplifies Eq. (19) to

$$
\frac{\partial P}{\partial y}=-\frac{3 y}{2 u^{2} t} P
$$

whose solution is the Gaussian of Eq. (15), but with $\langle n\rangle=(u t)^{2 / 3}$. The value $u=\sqrt{3 D}$ is determined by substituting $\langle n\rangle=(u t)^{2 / 3}$ in Eq. (18) and balancing the subdominant terms. To summarize, a Gaussian fitness distribution holds in both the critical and supercritical cases with the fitness distribution peaked at

$$
\langle n\rangle= \begin{cases}(3 D)^{1 / 3} t^{2 / 3} & \text { critical case } \\ V t & \text { supercritical case. }\end{cases}
$$

The age distribution in the critical case is obtained similarly to the supercritical case. The asymptotics of
$C_{n}(a, t)$ is again given by a form similar to Eq. (16), which gives $C(a, t)=\gamma N^{2} e^{-\gamma N a}$ after summing over $n$. Hence the average age is $B^{-1}$, as in Eq. (5).

While our discussion is based on a population dynamics with an age-independent mortality rate, this assumption does not substantially affect our main results. The crucial point is that old age is unattainable within our model. In the critical and supercritical cases, this is due to death by increased competition among fit individuals, while in the subcritical case, age is limited by the deleterious mutational bias. Thus for a more realistic mortality rate which increases with age, similar fitness and age dynamics to those outlined here would still result [9].

In summary, in our population dynamics model, the average fitness grows linearly in time when advantageous mutations are more likely and the fitness approaches a steady value when deleterious mutations are favored. In spite of this fitness evolution, the average age of the population always reaches a steady state. Intriguingly, this average age is a decreasing function of the average population fitness. This paradoxical behavior arises because competition becomes keener as the population becomes fitter.

We gratefully acknowledge partial support from NSF grant DMR9632059 and ARO grant DAAH04-96-1-0114.

[1] J. D. Murray, Mathematical Biology (Springer-Verlag, New York, 1989).

[2] B. Charlesworth, Evolution In Age-Structured Populations (Cambridge, UK, 1980).

[3] L. Tsimring, H. Levine, and D. Kessler, Phys. Rev. Lett. 76, 4440 (1996); D. A. Kessler, H. Levine, D. Ridgway, and L. Tsimring, J. Stat. Phys. 87, 519 (1997); D. Ridgway, H. Levine, and D. A. Kessler, J. Stat. Phys. 90, 191 (1998).

[4] P. M. C. de Oliveira, S. M. de Oliveira, A. T. Bernardes, D. Stauffer, Physica A 262, 242 (1999).

[5] An general discussion of related models is given by S. M. de Oliveira, P. M. C. de Oliveira, D. Stauffer, Evolution, Money, War, and Computers: Non-Traditional Applications of Computational Statistical Physics (Teubner Verlag, Stuttgart, 1999).

[6] T. J. P. P. Penna, J. Stat. Phys. 78, 1629 (1995).

[7] M. Ya. Azbel, Proc. Roy. Soc. London B, 263, 1449 (1996).

[8] R. M. Nisbet and W. S. C. Gurney, Modelling Fluctuating Populations (John Wiley \& Sons, 1982).

[9] W. Hwang, P. L. Krapivsky, and S. Redner, in preparation. 